\title{
THE BIRTH OF TWIN POINCARÉ-BIRKHOFF CHAINS NEAR 1:3 RESONANCE
}

\author{
J.P. VAN DER WEELE and T.P. VALKERING \\ Center for Theoretical Physics, University of Twente, P.O. Box 217, 7500 AE Enschede, \\ The Netherlands
}

H.W. CAPEL and T. POST

Institute for Theoretical Physics, University of Amsterdam, Valckenierstraat 65, 1018 XE Amsterdam, The Netherlands

Received 14 June 1988

\begin{abstract}
For a typical area-preserving map we describe the birth process of two twin PoincaréBirkhoff chains, i.e. two rings consisting of center points alternated by saddles, wound around an elliptic fixed point. These twin chains are not born out of the elliptic fixed point, but in the plane, from an annular region where the rotation number has a rational extremum. This situation generically occurs near a $1: 3$ resonance. We find that the birth of two twin PB chains in such an annular region requires first the birth of two "dimerized" chains of saddle-center pairs, by a tangent bifurcation. The transition from two dimerized chains to two PB chains involves the breakup of homoclinic saddle connections and the formation of heteroclinic connections; it amounts to the reconnection phenomenon of Howard and Hohs.

Our results can be regarded as a supplement to the Poincaré-Birkhoff theorem, for the case that the twist condition is not satisfied.
\end{abstract}

\section{Introduction}

Over the years, area-preserving maps with one variable parameter have been studied extensively, as representations of Hamiltonian systems with two degrees of freedom $[1,2]$. Most of these maps are non-integrable and show a rich dynamical structure. In a previous paper [3] we have described the squeeze effect, the collapse of the stable island around an elliptic fixed point at the $1: 3$ resonance. In this paper we will discuss a phenomenon which is linked to this squeeze effect: the birth of two twin Poincare-Birkhoff chains, when the rotation number has a rational extremum outside the elliptic fixed point. An early version of the present paper can be found in ref. [4].

Poincaré-Birkhoff chains (or PB chains for short) are very common in area-preserving maps. As a typical example let us consider the conservative 
Hénon map:

$$
\left\{\begin{array}{l}
x_{n+1}=2 C x_{n}+2 x_{n}^{2}-y_{n}, \\
y_{n+1}=x_{n}
\end{array}\right.
$$

which has an elliptic fixed point in the origin $(x, y)=(0,0)$ for all parameter values $1>\mathrm{C}>-1$. In fig. 1a we have shown the neighbourhood of the fixed point for $C=0.25$, exhibiting a typical PB chain consisting of five elliptic points alternated by five hyperbolic points, which together form two orbits of period 5 . Every elliptic point is surrounded by a small island, which is embraced by the two heteroclinic connections between the neighbouring hyperbolic points.

Apart from this PB chain the elliptic fixed point in the origin is surrounded
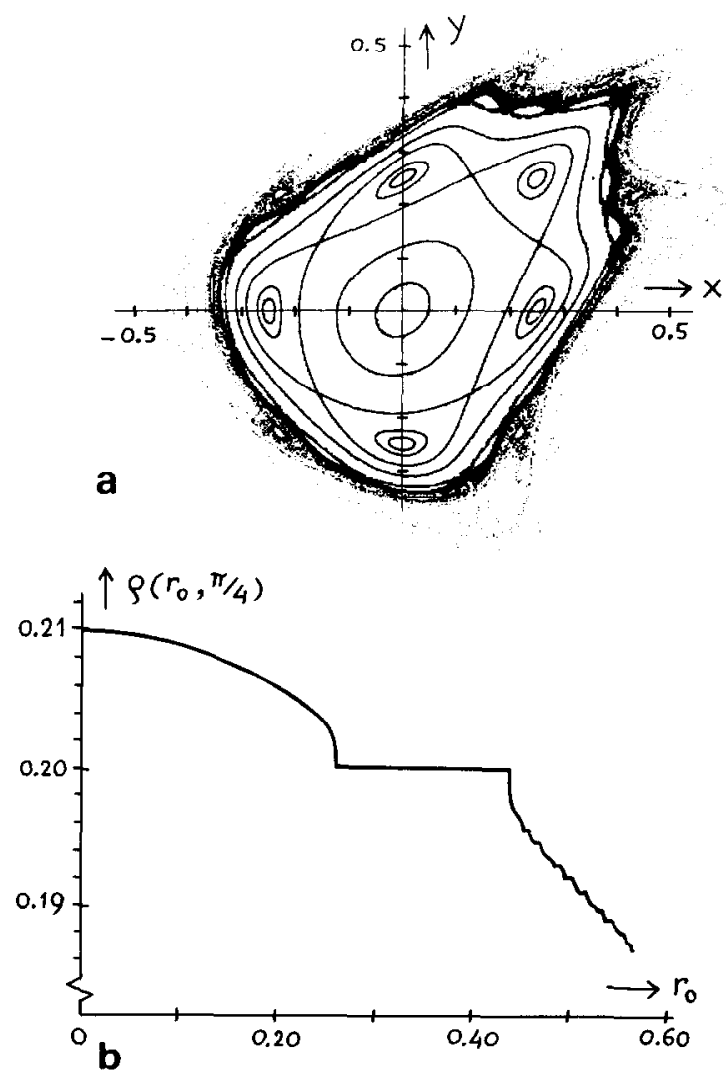

Fig. 1. (a) Several orbits in the neighbourhood of the origin for the Hénon map (1) at $C=0.25$. We see a Poincaré-Birkhoff chain consisting of five centers alternated by five saddles. (b) The rotation number $\rho\left(r_{0}, \pi / 4\right)$ versus $r_{0}$ for the situation depicted in fig. 1a. The large plateau at $\rho=\frac{1}{5}$ corresponds to the Poincaré-Birkhoff chain. 
by many more orbits which stay close to it, and which together constitute a stable island. All these orbits rotate around the origin and can be characterized by a rotation number $\rho$, which is defined as follows:

$$
\rho\left(r_{0}, \varphi_{0}\right)=\lim _{N \rightarrow \infty} \frac{1}{2 \pi N} \sum_{n=1}^{N}\left(\varphi_{n} \cdots \varphi_{n-1}\right) \quad \text { with } 0 \leqslant \varphi_{n}-\varphi_{n-1}<2 \pi,
$$

where $(r, \varphi)$ are the polar coordinates of $(x, y)$. The rotation number gives the average fraction of a revolution that is made per iteration, for an orbit starting at $\left(r_{0}, \varphi_{0}\right)$. By definition we have $0 \leqslant \rho\left(r_{0}, \varphi_{0}\right)<1$. In fig. $1 \mathrm{~b}$ we have shown $\rho\left(r_{0}, \varphi_{0}\right)$ as a function of $r_{0}$, with $\varphi_{0}=\pi / 4$ fixed, for the situation of fig. 1a.

A few general comments about this curve are in order. When $\rho\left(r_{0}, \varphi_{0}\right)$ has an irrational value, the orbit goes round and round without ever hitting exactly upon its starting point again. In that case we can get a densely filled curve (a torus) without any gaps, or a Cantor-type curve with innumerable gaps. Both are called quasiperiodic $[5,6]$. In between these curves we also find rational values of $\rho\left(r_{0}, \varphi_{0}\right)$, that is $\rho\left(r_{0}, \varphi_{0}\right)=p / q$, with $p$ and $q$ coprime integers. In that case the orbit is either periodic with period $q$, or it is a satellite of such an orbit (i.e. any bounded orbit with the same rotation number). The periodic orbit is either elliptic or hyperbolic. If it is elliptic, the satellite region consists of the islands around the periodic points. This explains the plateaus in the plot of fig. $1 b$. In theory, one expects a plateau at every rational value $p / q$, but in practice one can only see the plateaus with $q$ not too large, because the width of the plateaus decreases rapidly for growing $q$. If the orbit is hyperbolic, it is a little bit more complicated to point out the satellite region. But the stable manifolds of each of the saddle points certainly belong to it, and one may assume that these contaminate the whole region embedded by the (tangled) saddle connections.

Now, as $C$ is decreased one observes that the whole curve of rotation numbers is pushed upward (see also ref. [3]). And every time the maximum of this curve hits upon a new rational valuc $p / q$, onc or more periodic orbits with period $q$ will be born.

For the parameter intervals $1 \geqslant C \geqslant-0.25$ and $-0.5 \geqslant C \geqslant-1$ the $\rho\left(r_{0}, \pi /\right.$ 4) curve has its maximum at $r_{0}=0$. This maximum increases for decreasing $C$ according to

$$
\rho\left(r_{0}=0\right)=\frac{1}{2 \pi} \arccos C .
$$

So for $C$ values within these intervals the new PB chains are born at the origin, at values $C_{p / q}=\cos (2 \pi p / q)$, to be pushed outward for decreasing $C$. This type of birth (bifurcation from an elliptic fixed point) is well understood $[7,8]$. 
But for the parameter interval $-0.25>C>-0.50$ the curve of $\rho\left(r_{0}, \pi / 4\right)$ has its maximum outside the origin [3,4]. This is illustrated in fig. 2 , where we have shown the situation for $C=-0.40$. It should be noted that the values of $\rho$ in this figure lie just below $\frac{1}{3}$. This is not a coincidence, since the maximumoutside-the-origin typically occurs as a prelude to the $1: 3$ resonance.

In fact, for the map (1) the maximum value at $C=-0.25$ (still at the origin) is $\rho=(1 / 2 \pi) \arccos (-0.25)=0.2902$. For decreasing $C$ the maximum moves away from the origin, and increases at the same time, until it reaches the value $\rho=\frac{1}{3}$ at $r_{0}=0.2929$, that is at $(x, y)=(0.2071,0.2071)$. This happens at $C=1-\sqrt{2}=-0.4142$. At this instant two 3 cycles are born by a tangent bifurcation, one hyperbolic and one elliptic. If $C$ is decreased further the points of the hyperbolic 3 cycle move towards the origin, squeezing its stable island, and bringing the maximum of the $\rho$ curve back to the origin. At $C=\frac{1}{2}$ the
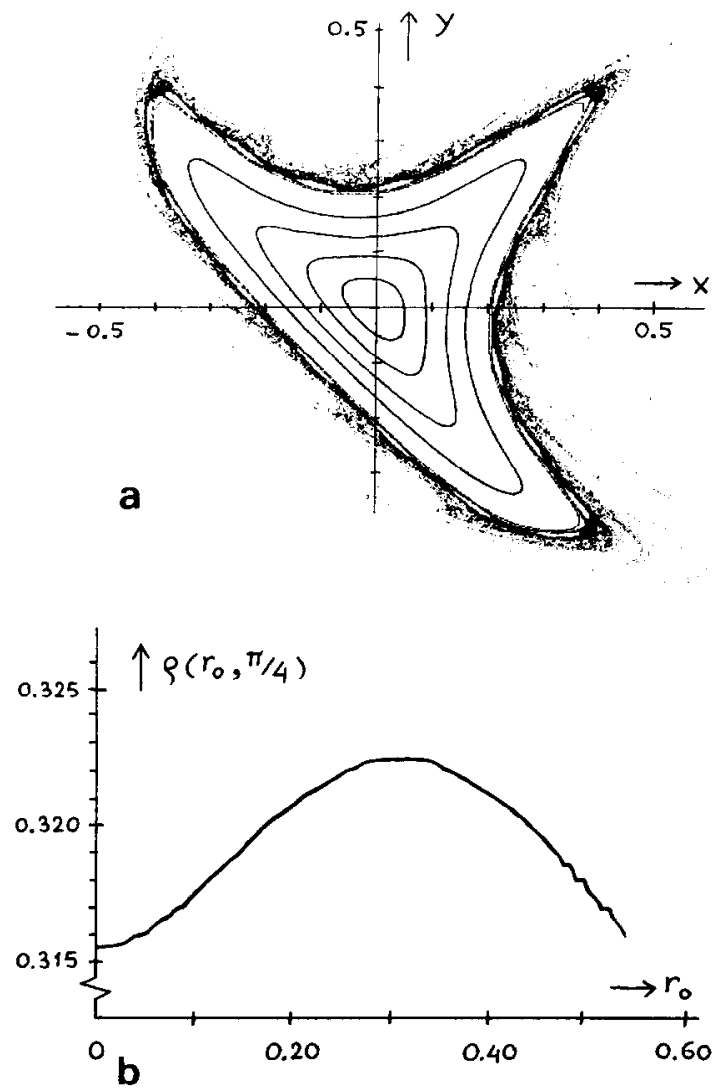

Fig. 2. (a) Several orbits in the neighbourhood of the origin for the Hénon map (1) at $C=-0.40$. (b) The rotation number $\rho\left(r_{0}, \pi / 4\right)$ versus $r_{0}$ for the situation depicted in fig. $2 \mathrm{a}$. 
hyperbolic 3 cycle coincides with the origin ( $1: 3$ resonance), and for $C<-\frac{1}{2}$ it emerges again; in its wake a new island appears, now with $\rho>\frac{1}{3}$. This means that at $C=-\frac{1}{2}$ the maximum of $\rho$ breaks through the value $\frac{1}{3}$ at the origin, and for decreasing $C$ the maximum remains at the origin. This has also been described in ref. [3].

So the periodic orbits with $0.2902<\rho<\frac{1}{3}$ are both outside the origin, every time the maximum hits upon a new rational value $p / q$. And immediately after the maximum has pierced through this value there must be two PB chains with $\rho=p / q$, one at each side of the maximum. This is because at both sides the twist condition of the Poincaré-Birkhoff theorem (see section 3 ) is satisfied. For decreasing $C$, the inner PB chain moves inward until it dies at the origin (at $C=\cos (2 \pi p / q))$, and the outer PB chain moves outward until it falls off the island.

It is the purpose of this paper to describe the birth process of these twin PB chains. That is, we are going to investigate what happense between the moment when the maximum hits the value $p / q$ for the first time, and the moment when the maximum pierces through this value.

In section 2.1 we present a "movie" of the birth process of two twin PB chains with $\rho=\frac{3}{10}$, for the Hénon map (1). The birth process involves two dimerized periodic chains, different from the usual PB chains. The subsequent transition between the dimerized chains and the twin PB chains turns out to amount to a reconnection phenomenon which has been observed before by Howard and Hohs [9], who treated it on the basis of a radial twist mapping. In section 2.2 we comment upon the relevance of this birth process in connection with the $1: 3$ resonance. Finally, in section 3 we argue that our results can be regarded as a supplement to the Poincaré-Birkhoff theorem.

\section{The birth process}

\subsection{A typical example}

In this section we present a typical example of the birth process of two twin PB chains. As remarked in the introduction, the rotation numbers for the map (1) show an out-of-the-origin maximum for the parameter interval $-\frac{1}{4}>C>$ $-\frac{1}{2}$. The corresponding range of rotation numbers is, via the relation (3),

$$
0.2902153 \ldots<\rho<\frac{1}{3} \text {. }
$$

That is, if $C$ is decreased from $C=-\frac{1}{4}$ to $-\frac{1}{2}$ the maximum takes on all possible values between $0.2902153 \ldots$ and $\frac{1}{3}$. Among these is the rational value 
$\frac{3}{10}$, which happens to be the maximum value in an interval close to $C \approx$ -0.3063 . We will concentrate on this example. Of course, we could also have taken other examples, for instance the birth of the twin PB chains with $\rho=\frac{4}{13}=0.3077$. This takes place close to $C \approx-0.345$, when the maximum lies at $r_{0} \approx 0.22$. Or we could have taken the birth of the twin PB chains with $\rho=\frac{5}{16}=0.3125, \rho=\frac{5}{17}=0.2941$, etc. All these yield the same kind of pictures.

Figs. 3-7 show five stages of the birth process of the $\frac{3}{10}$ chains. Each picture is accompanied by a plot of the rotation number along the line $\varphi_{0}=\pi / 4$ (or $x=y$ ), which is a symmetry line for the map (1).

In fig. 3 , at $C=-0.306286$, we see the situation just before the maximum of $\rho\left(r_{0}, \pi / 4\right)$ reaches the value $\frac{3}{10}$. The maximum has the form of a sharp peak, indicating that the $\frac{3}{10}$ orbit which is going to be formed will be very narrow at the intersection with the $x=y$ line. This is sustained by the picture of the $x y$-plane, which shows how orbits in the neighbourhood of the maximum begin to meander, creating two blobs to the left and to the right of the $x=y$ line, and squeezing the orbits together at the $x=y$ line itself. If we would have cut through one of the blobs (there are 10 of them in total) we would have measured a broad maximum of $\rho$.

In fig. 4 , at $C=-0.306288$, the maximum of $\rho\left(r_{0}, \pi / 4\right)$ has reached the value $\frac{3}{10}$. We see that inside the blobs there have been tangent bifurcations creating saddle-center pairs, which together from two periodic $\frac{3}{10}$ orbits, one elliptic and one hyperbolic. A plot of $\rho\left(r_{0}, \varphi_{0}\right)$ through one of the blobs would yield a flat plateau as maximum of $\rho$.

Each saddle point has one homoclinic connection around the island of the nearby center point, and one heteroclinic connection with each of its two
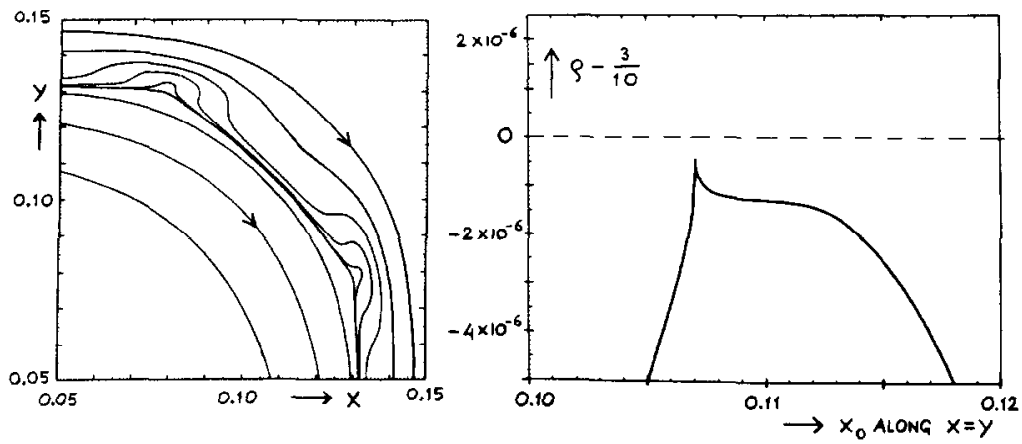

Fig. 3. Detail of the stable island around the origin, for the map (1) at $C=-0.306286$. The arrows indicate the motion under $T^{10}$, i.e. the map (1) iterated 10 times. All the curves in this picture have rotation numbers that are (slightly) smaller than $\frac{3}{10}$. Also shown is a plot of the rotation number $\rho$ along the line $x=y$. To make the two figures correspond as clearly as possible we have plotted $\rho$ as a function of $x_{0}=r_{0} / \sqrt{2}$, instead of $r_{0}$. 

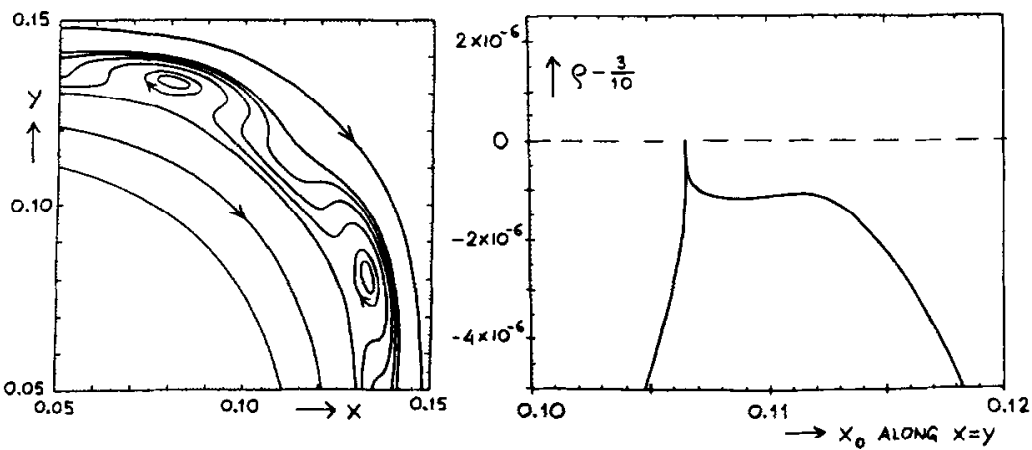

Fig. 4. The same region as in fig. 3, but now at $C=-0.306288$. Inside the inner curls of the meander we have saddle-center pairs. Also shown is the plot of $\rho$ along the line $x=y$. The peak in this plot indicates the presence of a heteroclinic saddle connection.

neighbour saddles. Clearly, the two periodic orbits do not form a PB chain, which has two heteroclinic connections between neighbouring saddles. Rather, they form a chain of saddle-center pairs, with only one heteroclinic connection between neighbouring saddles. We call this a dimerized chain.

One heteroclinic connection crosses the $x=y$ line, yielding the sharp peak in $\rho\left(r_{0}, \pi / 4\right)$. On the scale of our figure this peak cannot be distinguished from a cusp. In reality it cannot be a cusp, however, since the heteroclinic tangle has a small but finite width. To the right of this peak we see a broad hump rising towards $\frac{3}{10}$, anticipating the birth of a saddle-center pair on the line $x=y$. Indeed, the developing meanders in the $x y$ plane also hint in this direction.

In fig. 5 , at $C=-0.306290$, this birth is a fact. Now, there are two dimerized chains, one on the inside of the meanders and one on the outside. These
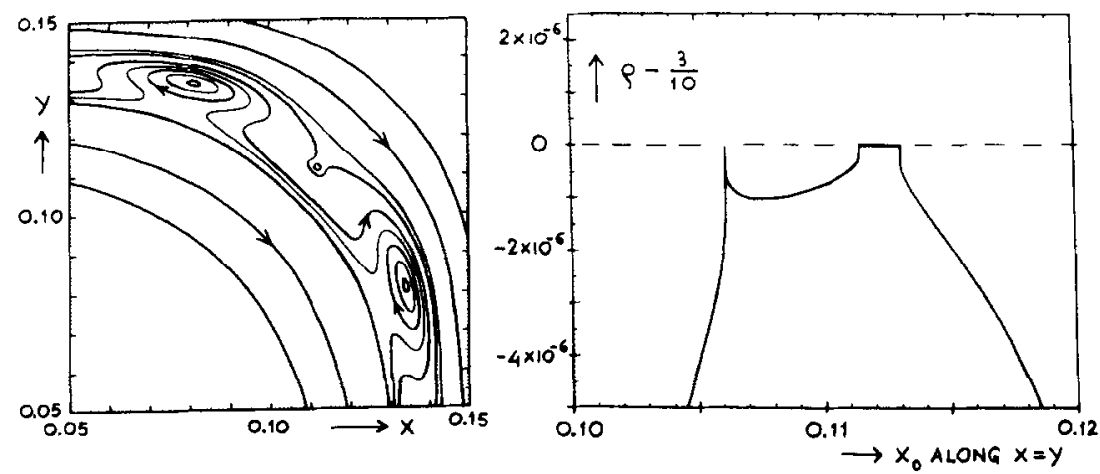

Fig. 5. The same region as in figs. 3 and 4 , but now at $C=-0.306290$. We have saddle-center pairs in the inner curls of the meander as well as in the outer curls. Also shown is the plot of $\rho$ along the line $x=y$. The peak indicates a heteroclinic saddle connection, and the plateau indicates the saddle-center pair. 
meanders are quasiperiodic orbits with $\rho<\frac{3}{10}$. They are now on both sides surrounded by $\frac{3}{10}$ orbits, as is also evident from the plot of $\rho\left(r_{0}, \pi / 4\right)$ along the $x=y$ line. The peak indicates the heteroclinic saddle connection of the inner dimerized chain, and the plateau corresponds to the saddle-center pair of the outer dimerized chain. The slopes of $\rho\left(r_{0}, \pi / 4\right)$ near the peak and the edges of the plateau are very steep.

Note that the two dimerized chains together contain two elliptic and two hyperbolic orbits of period 10. This is just the number needed for the creation of two PB chains; only the points should be re-arranged. This will be the next step in the birth process.

In fig. 6 , at $C=-0.306297$, we are extremely close to the marginal situation. The saddles and the centers are on the verge of being re-arranged into two PB chains. The plot of $\rho\left(r_{0}, \pi / 4\right)$ along the $x=y$ linc shows an uninterrupted plateau, extending from the heteroclinic saddle connection, through the island (which is responsible for almost all of the width of the plateau) to the saddle. In this plot there is no visible trace of a meander anymore, though in the $x y$-plane we can still discern one meandering curve which is almost degenerated to an array of triangles.

Finally, in fig. 7, at $C=-0.306299$, the two newly arranged PB chains have moved apart, and between them we see a closed curve with rotation number $\rho>\frac{3}{10}$. This is also clear from the plot of $\rho\left(r_{0}, \pi / 4\right)$ along the $x=y$ line. The saddle region of the outer PB chain, which lies on the line $x=y$, has negligible width and appears only as a point in this plot. The plateau is due to the island of the inner chain. A plot of $\rho\left(r_{0}, \varphi_{0}\right)$ for other values of $\varphi_{0}$ will in general show two plateaus at the value $\frac{3}{10}$, separated by a region where $\rho$ has a
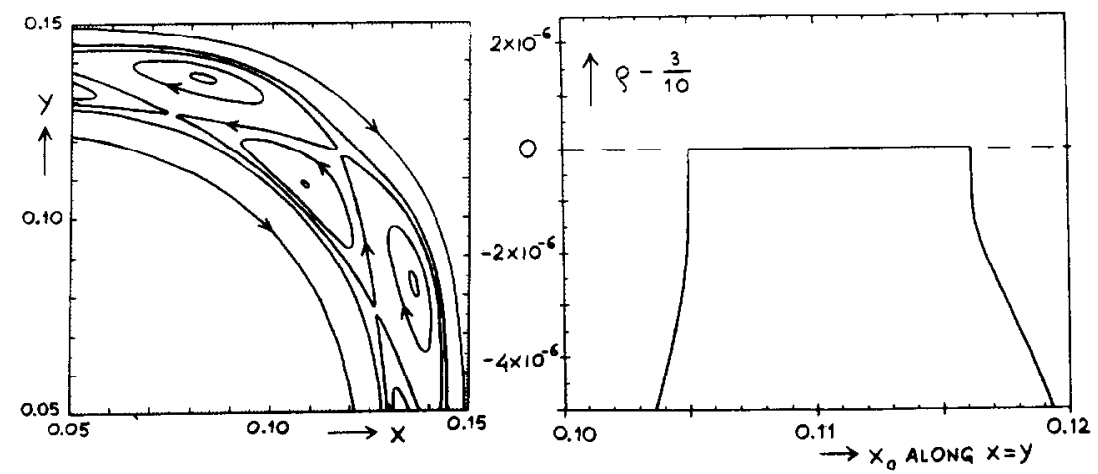

Fig. 6. The same region as in figs. $3-5$, but now at $C=-0.306297$. It illustrates the marginal situation; at this moment the saddles and centers are being rearranged to form two PoincaréBirkhoff chains. Also shown is the plot of $\rho$ along the line $x=y$. It exhibits an uninterrupted plateau. 


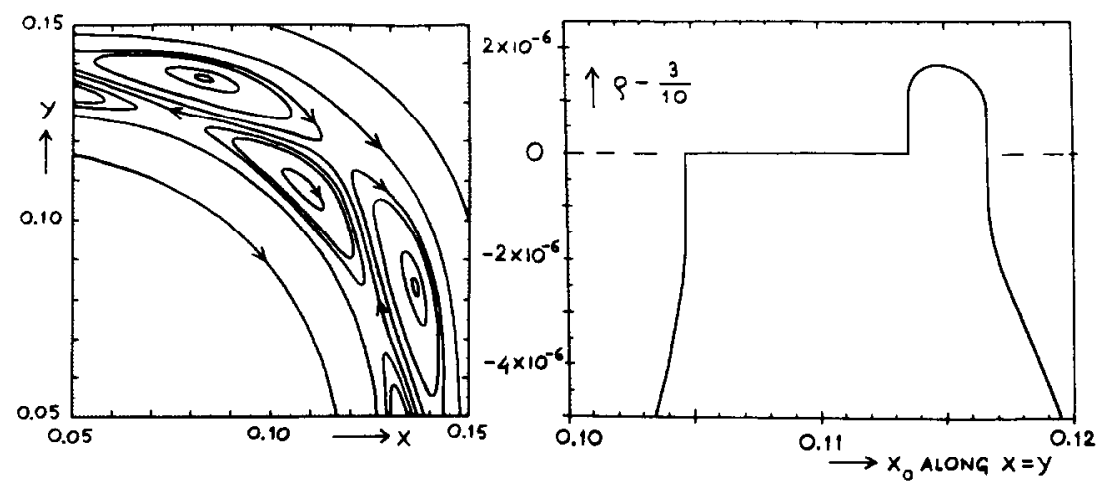

Fig. 7. The same region as in figs. 3-6, but now at $C=-0.306299$. The two Poincaré-Birkhoff chains have moved apart, and between them one sees a curve with rotation number $\rho>\frac{3}{10}$. The plot of $\rho$ along the line $x=y$ shows clearly that the maximum has finally pierced through the value $\frac{3}{10}$.

maximum. (One of these plateaus is reduced to a point if we cut exactly through a saddle.)

In conclusion, we have seen that the birth of two twin PB chains requires the birth of two dimerized chains first. In fig. 8 we have illustrated the successive stages of the process.

First one dimerized chain appears, then a second, and after that the centers

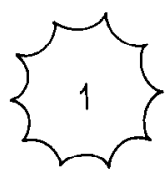<smiles>CC1(C)C(=O)C(=O)C(=O)C(=O)C(=O)C(=O)C1(C)C</smiles>
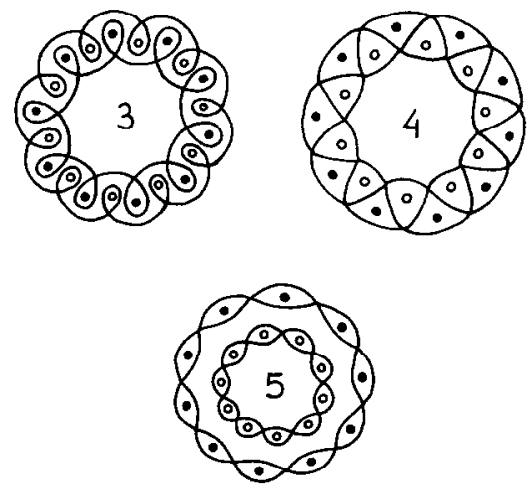

Fig. 8. Schematic representation of the five stages of the birth process of two twin PB chains, corresponding to figs. 3-7. 1. Preparation; 2 . one dimerized chain; 3 . two dimerized chains; 4. marginal situation; 5. two PB chains. 
of the two chains are interchanged due to the breakup of the homoclinic connections and the formation of new heteroclinic ones. This yields two PB chains.

The birth scenario found above turns out to be very similar to the one presented in ref. [9], to describe the reconnection phenomenon in a radial twist map with a non-monotonic twist function: $x_{n+1}=x_{n}-K \sin \theta_{n}, \theta_{n+1}=\theta_{n}+$ $f\left(\alpha, x_{n+1}\right)$ with $f(\alpha, x)=x-\alpha x^{2}$. The main difference is that the scenario for this map does not contain a regime with only one dimerized chain. Furthermore, in ref. [9] the relation to the rotation number and the $1: 3$ resonance was not discussed, nor the connection with the Poincaré-Birkhoff theorem.

\subsection{A few comments}

The birth of two twin PB chains is by no means unusual. In fact, in an earlier paper [3] we found that it is a necessary prelude to the squeeze effect, which occurs at the $1: 3$ resonance in every area-preserving map with a non-vanishing quadratic part (and sometimes also at the 1:4 resonance). Thus, its implications can be observed in many physical systems. One example is the beambeam interaction in a storage ring, in which the transition from two dimerized chains to two PB chains has indeed been observed by Gerasimov et al.; see figs. $15 \mathrm{a}$ and $15 \mathrm{~b}$ of ref. [10]. Each chain consists of 10 islands and 10 saddles, just as in our example with $\rho=\frac{3}{10}$. Their pictures show that it is quite a macroscopic effect, too.

The link with the $1: 3$ resonance means that the twin births will generally occur for values of $\rho$ close to $\frac{1}{3}$, but the exact range of rotation numbers is not the same for every map. For the generalized Hénon map

$$
\left\{\begin{array}{l}
x_{n+1}=2 C x_{n}+a x_{n}^{2}+b x_{n}^{3}-y_{n}, \\
y_{n+1}=x_{n}
\end{array}\right.
$$

(taken from ref. [3]), we have twin births for

$$
\frac{1}{2 \pi} \arccos \left(C_{0}\right)<\rho<\frac{1}{3},
$$

where

$$
C_{0}=\frac{1}{4}+\gamma-\sqrt{\gamma^{2}+\gamma+\frac{9}{16}} \quad \text { with } \gamma=a^{2} / 3 b .
$$

Note that for $a \neq 0$ and $b=0$ (i.e. $\gamma=\infty$ ) we have $C_{0}=-\frac{1}{4}$, the same value as for the quadratic Hénon map (1), which has $a=2, b=0$. On the other hand, 
for $a=0$ and $b \neq 0$ (i.e. $\gamma=0)$ we get $C_{0}=-\frac{1}{2}$ and $(1 / 2 \pi) \arccos \left(C_{0}\right)=\frac{1}{3}$. In this (non-generic) cubic case the range of rotation numbers as given by eq. (6) is reduced to nothing. This is directly related to the fact that in this case we do not have a squeeze effect at the $1: 3$ resonance [3].

Eq. (6) tells us that the lower bound of the range of rotation numbers varies with the particular map under consideration, but the upper bound is always $\frac{1}{3}$. This can be explained as follows. The birth process of two PB chains necessarily requires two dimerized chains. But there is no strict law which demands that after the first dimerized chain there will always come a second. In fact, for $\rho=\frac{1}{3}$ only one dimerized chain appears; for the map (1) this occurs at the value $C=1-\sqrt{2}=-0.4142$. We have described this in detail in ref. [3]. In that case, we can neither get two PB chains, nor can any closed curve with $\boldsymbol{\rho}>\frac{1}{3}$ come between the saddles and the centers of the dimerized chain. This is because on both sides of a such a hypothetical curve the twist condition of the Poincaré-Birkhoff theorem (see section 3 ) would be satisfied, and consequently we should have a PB chain with $\rho=\frac{1}{3}$ on both sides. But there are simply not enough center and saddle points to accommodate two PB chains. Thus, $\rho=\frac{1}{3}$ is the upper bound for the range of rotation numbers associated with twin births.

\section{Connection with the Poincaré-Birkhoff theorem}

The Poincaré-Birkhoff theorem $[1,2,11-13]$ states that for area-preserving twist maps, given integers $p$ and $q$ with $q$ positive, there are at least two periodic orbits satisfying

$$
\left\{\begin{array}{l}
r_{q}=r_{0}, \\
\varphi_{q}=\varphi_{0}+2 \pi p .
\end{array}\right.
$$

In other words there are at least two periodic orbits with rotation number $p / q$. If these orbits are isolated then, according to the theorem, one of them is elliptic and the other one is hyperbolic, together forming a PB chain.

The twist condition in this theorem requires that the annular region on which the rational value $\rho=p / q$ occurs is bounded on one side by an orbit with $\rho<p / q$ and on the other side by an orbit with $\rho>p / q$. As we have seen, this condition is not always satisfied. On the contrary, for a substantial range of parameter values every periodic orbit (being created in an annular region around the elliptic fixed point) is born from a non-twist situation. In that case the periodic orbits are born as dimerized chains, via a tangent bifurcation. If 
only one dimerized chain appears we have the squeeze effect as described in ref. [3]. Otherwise, two dimerized chains appear, either one after the other (as for the Hénon map described in the present paper) or simultaneously (as for the twist map in ref. [9]). The two chains will, upon a change of parameter value, be rearranged in the form of twin $\mathrm{PB}$ chains, at the very moment that the twist condition is going to be satisfied. Indeed, these PB chains could never be created if the dimerized chains would not pave the road for them.

So we see that the birth mechanism described in this paper provides a supplement to the Poincaré-Birkhoff theorem, in the case that the rotation number has a rational extremum outside the origin.

\section{Acknowledgements}

One of the authors (JPvdW) is indebted to D.F. Escande en R.S. MacKay for useful comments.

\section{References}

[1] Hamiltonian Dynamical Systems, a reprint selection, R.S. MacKay and J.D. Meiss, eds. (Adam Hilger, Bristol, 1987).

[2] R.S. MacKay, Introduction to the dynamics of area-preserving maps, in: Proc. US Particle Accelerator School, M. Month, ed. (SLAC, 1985).

[3] J.P. van der Weele, H.W. Capel, T.P. Valkering and T. Post, Physica A 147 (1988) 499.

[4] J.P. van der Weele, Scaling and squeezing in nonlinear dynamics, thesis, University of Amsterdam (1987), chap. 6.

[5] J.N. Mather, Topology 21 (1982) 457.

[6] S. Aubry and P.Y. Le Daeron, Physica D 8 (1983) 381.

[7] K.R. Meyer, Trans. Am. Math. Soc. 149 (1970) 95, reprinted in ref. [1]; Lecture Notes in Mathematics, vol. 468 (1975), p. 62.

[8] J.M. Greene, R.S. MacKay, F. Vivaldi and M.J. Feigenbaum, Physica D 3 (1981) 468, reprinted in ref. [1].

[9] J.E. Howard and S.M. Hohs, Phys. Rev. A 29 (1984) 418.

[10] A. Gerasimov, F.M. Israilev, J.L. Tennyson and A.B. Temnykh, Springer Lecture Notes in Physics, vol. 247 (1986), p. 154, reprinted in ref. [1].

[11] H. Poincaré, Rendiconti del Circolo Mat. di Palermo 33 (1912) 375.

[12] G.D. Birkhoff, Trans. Am. Math. Soc. 14 (1913) 14; Acta Math. 47 (1925) 297.

[13] M.V. Berry, Regular and irregular motion, in: Topics in Nonlinear Dynamics, S. Jorna, ed., AIPCP 46 (1978) 16, reprinted in ref. [1]. 